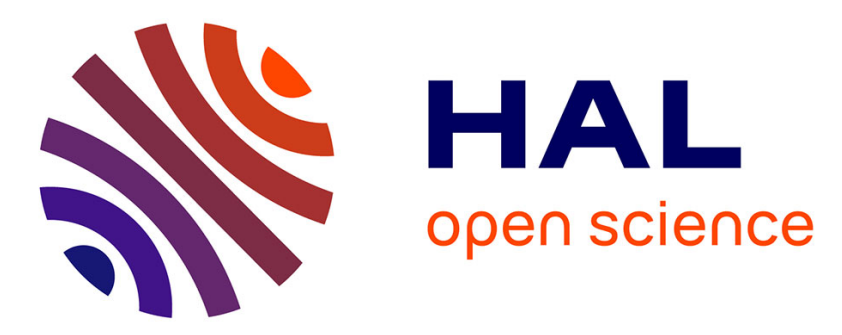

\title{
A Comprehensive Assessment of the Short-Term Uncertainty of Grid-Connected PV Systems
}

Enrica Scolari, Lorenzo Reyes-Chamorro, Fabrizio Sossan, Mario Paolone

\section{To cite this version:}

Enrica Scolari, Lorenzo Reyes-Chamorro, Fabrizio Sossan, Mario Paolone. A Comprehensive Assessment of the Short-Term Uncertainty of Grid-Connected PV Systems. IEEE Transactions on Sustainable Energy , 2018, 9 (3), pp.1458-1467. 10.1109/TSTE.2018.2789937 . hal-02108863

\section{HAL Id: hal-02108863 \\ https://hal.science/hal-02108863}

Submitted on 24 Mar 2021

HAL is a multi-disciplinary open access archive for the deposit and dissemination of scientific research documents, whether they are published or not. The documents may come from teaching and research institutions in France or abroad, or from public or private research centers.
L'archive ouverte pluridisciplinaire HAL, est destinée au dépôt et à la diffusion de documents scientifiques de niveau recherche, publiés ou non, émanant des établissements d'enseignement et de recherche français ou étrangers, des laboratoires publics ou privés. 


\title{
A Comprehensive Assessment of the Short-Term Uncertainty of Grid-Connected PV Systems
}

\author{
E. Scolari, Student Member, IEEE, L. Reyes-Chamorro, Member, IEEE, F. Sossan, Member, IEEE, \\ M. Paolone, Senior Member, IEEE
}

\begin{abstract}
One of the key aspects enabling the bulk integration of photovoltaic (PV) resources into the power grid is the shortterm prediction of the maximum available power (from $100 \mathrm{~ms}$ to 5 minutes), and the quantification of the associated uncertainties. This is beneficial for the definition of robust control strategies able to account for the stochastic nature of this energy resource. We propose and validate a comprehensive method to assess the overall PV power uncertainties, even at operating conditions different from the maximum power point (MPP), i.e., to consider when power curtailment strategies are adopted on a controllable PV plant. The proposed gray-box modeling includes physical and data-driven sub-models that rely on measurements of the PV currents, voltages, and the module temperature, information normally available to the PV plant operator. Furthermore, we identify which sub-model is the most critical in terms of uncertainty, for different forecast horizons. Experimental results analyze the ability of the method to guarantee the target coverage probability while accounting for the uncertain nature of the PV resource. We show how modelling and forecasting information can be used to express the PV plant behaviour to a grid controller responsible for the safe operation of a microgrid.
\end{abstract}

Index Terms-Photovoltaic modeling, gray-box modeling, solar forecast, uncertainty assessment, microgrid.

The increasing proportion of distributed generation (DG) in the power generation mix requires redefining conventional practices to mitigate the impact of stochastic resources like photovoltaic (PV) systems [1]. The main issues are related to the operational limits of the local grid, in terms of quality of supply and line congestions [2]-[4]. In particular, the PV power output at low level of aggregation can contain variations on short time scales (lower than 1 second) due to high-frequency irradiance fluctuations [5]. These short-term dynamics can cause violations of the quality requirements for electrical networks like voltage flickers [6]. To respond to these issues, PV inverters are generally used for droopbased voltage regulation by controlling the reactive power injections [7]. However, since power distribution networks are characterized by lines parameters with large $R / X$ (ratio of the components of their longitudinal impedances), there is generally the need of coupling the reactive power control with active power curtailment (APC) strategies [8]-[10]. The main concern related to simple APC strategies is that the active power curtailment might not be the most favorable solution if, for example, other resources (e.g. storage systems) are available in the grid. In this respect, there is an increasing attention in the literature towards new control approaches that are able to explicitly account for the aforementioned

This research received funding from the Swiss Competence Center for Energy Research (FURIES) and by the SNSF NRP70 Energy Turnaround. technical issues while intelligently exploiting the flexibility of all the available resources [11], [12]. For the case of microgrids, their limited geographical extension joined with the capability to function autonomously determine the need for sub-second time-scale control. In this context, several control strategies are presented in the literature [1]. In particular, two main approaches are followed i) distributed/decentralized and (ii) fully centralized. In the decentralized case, improved droop strategies that account for the internal state of the resources are proposed as a natural extension of consolidated control philosophies, (e.g. [13], [14]). Alternatively, droop-free methods based on multi-agents systems (MAS) are proposed, where agents exchange their objectives and uncertainties to reach a consensus and minimize a given shared cost function (e.g. [15], [16]). In the case of centralized approaches, a central controller is responsible for the solution of an optimal dispatch problem constrained by the grid operation limits. MAS are usually proposed also in the centralized case, [17], where a central agent receives messages from the followers, solves an overall problem, and sends the messages back to the followers. The knowledge of the PV short-term behavior and its uncertainties becomes a key factor to ensure the robustness of the mentioned control strategies, [18]. In this work, we propose a gray-box method to assess the overall PV plant flexibility and associated uncertainties at different forecast horizons, from $100 \mathrm{~ms}$ to $5 \mathrm{~min}$. The contributions of the work are the following. First, we describe how to accurately predict the one-step-ahead AC maximum available power, integrating a model for the PV modules, a model for the converter, and a probabilistic short-term forecasting tool. The sub-models are selected to be of easy reproducibility, and the methodology needs few inputs: i) information from the PV module datasheet, ii) real-time measurements of the PV plant voltages and currents, and iii) a temperature sensor. The available literature generally focuses on the individual submodel accuracy, e.g. [19], [20], while we here evaluate the overall chain that allows obtaining the $\mathrm{AC}$ maximum power. For different forecast horizons and, for the first time, for different levels of curtailed energy, we identify which submodel is the most critical. The validation is made possible by a dedicated experimental setup that relies on a fully controllable converter, [21], which can track an external power reference of active and reactive power. Second, the uncertainty of the PV converter in tracking an external setpoint is accounted. Indeed, due to the internal characteristic of the converter (e.g. control loop), the implemented power injection can differ from the request. Finally, we show how the proposed methodology can 
be used to advertise the PV plant future behaviour to a central grid controller. This is relevant to allow model predictive control approaches to intelligently exploit the PV flexibility, aiding PV integration into the grid.

The work is structured as follows: Section I defines the problem, Section II describes the experimental setup used for the validation, Section III discusses the different blocks used to describe the PV behaviour and the related literature, Section IV shows the results of the uncertainty assessment. Finally, Section V shows how to design a PV agent, Section VI presents a further discussion on the methods, and Section VII draws the main conclusions.

\section{Problem Definition}

We consider a generic PV system (Fig. 1), with the following characteristics (i) it is always connected to the grid as a grid-feeding converter [22], (ii) it allows to track an external active/reactive power setpoint $u=\left(P^{*}, Q^{*}\right)$ from a Grid Controller (GC). The PV system has an inherent flexibility to modify its power operating conditions, namely the capacity of curtailing the generation of this resource, along with the possibility of controlling its reactive-power injection.

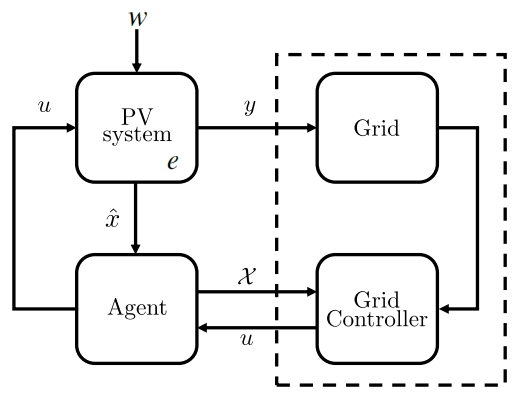

Fig. 1. Problem context. We consider a generic PV plant connected to the grid. The resource is equipped with an agent that can communicate with a generic grid controller. The dashed lines delineate exogeneous elements to the resource.

In order to allow the GC to exploit this flexibility, a dedicated agent is introduced. It continuously receives an updated state $\hat{x}$ from the PV system (e.g., the measured power value) and sends to the $\mathrm{GC}$ a feedback $\mathcal{X}$ that represents the expected one-step-ahead operational flexibility and uncertainty (see Section V). It is important to note that, since the converter can track external power setpoints, the measured AC power does not necessarily correspond to the maximum available one, and may not be representative of the production potential of the plant, that mostly depends on the external weather conditions $w$ (e.g., solar irradiance). Therefore, a physical model of the PV system is needed to reconstruct at each time-step the AC maximum available power, as detailed in Section III. These reconstructed power values are then used to train a time-seriesbased forecasting tool needed to predict the one-step-ahead $\mathrm{AC}$ maximum power. The uncertainty related to the delivered prediction is explained by both the stochasticity of the forecast and the PV/converter modeling errors. Furthermore, due to the converter internal operational constraints $e$, the external power reference $u=\left(P^{*}, Q^{*}\right)$ differs from the actual implemented power injection $y=(P, Q)$, this representing a further source of uncertainty. Consequently, a comprehensive uncertainty assessment is needed to account for these aspects: i) uncertainty on the one-step-ahead maximum available power computation, and ii) uncertainty on the setpoint tracking.

\section{EXPERIMENTAL SETUP}

We anticipate in this section the description of the experimental setup to introduce information about the observed state variables of the system we target to control. The PV system under test is detailed in Fig. 2. The power stage includes a PV-Side Converter (PVSC), that controls the power flow injected by the PV panels into the common DC-bus, and a Grid-Side Converter (GSC) responsible of controlling active and reactive power flows injected into the grid. We assume to have access to the measurements of the DC voltage, DC current, and average cell temperature of the PV array $(v, i, \theta)$, and to the voltages and currents phasors at the fundamental frequency components from the three-phase AC grid $(\bar{V}, \bar{I})^{1}$. The global irradiance on the PV plant $G$ could be measured by a sensor (e.g. pyranometer), but in this paper, it is inferred from the PV-array measurements as in [23], and detailed in Section III.

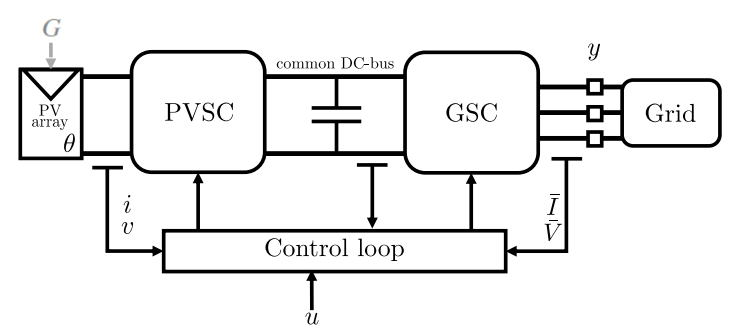

Fig. 2. Detailed view of the PV system.

The PV array consists in a $13 \mathrm{kWp}$ rooftop PV installation with 51 polycrystalline modules. The agent (Fig. 1) is implemented in a NI CompactRIO 9068 and communicates with the converter through a CAN bus interface. Currents and voltage measurements are acquired with a 16 bit ADC (NI-9215). Currents are measured with LEM LF 205-S transducers with an accuracy of $\pm 0.2 \%$. Voltages are measured with LEM CV 3-100 transducers with an accuracy of $\pm 0.5 \%$. The cell temperature is measured using a TSic303, a sensor with $\pm 0.5^{\circ} \mathrm{C}$ accuracy, installed on the rear surface of one panel and acquired by a NI sbRIO 9625 with a 16 bit ADC. All measurements are synchronized and sampled at $20 \mathrm{kHz}$. Since our analysis includes curtailment periods, a second equivalent PV system is used as a reference installation to obtain the maximum PV power output when the test plant is operated in non-MPPT mode. ${ }^{2}$. The equivalent behaviour of the two converters is experimentally compared by running both of

\footnotetext{
${ }^{1}$ These electrical quantities are commonly available in commercial PV converters, while the PV-array temperature can be easily measured.

${ }^{2}$ This reference PV system is needed only to assess the performance of the proposed method. The considered models are based on measurements from the tested PV system and, as later explained in the text, the presence of a reference system is not necessary to the final user.
} 
them in MPPT mode. The test is performed on an 11 days period characterized by mixed weather conditions (3 clearsky days, 2 overcast days, and 6 partly-cloudy days). In these conditions, the average absolute difference between the delivered AC active power values is less than $100 \mathrm{~W}$. Therefore we can assume that the behaviours of the two systems are comparable.

\section{Methods}

In this section we propose a method that, regardless of the PV system operating point, is able to i) provide a probabilistic forecast of the one-step-ahead maximum available power, and ii) determine the uncertainty on tracking a power setpoint. For short-term horizons (from sub-seconds to minutes), the first goal can be achieved by using time-series based forecasting methods, [18]. In particular, thanks to the increasing availability of electrical measurements, the use of direct forecast methods (that directly learn from historical power values) has recently gained interest, e.g. [24], [25]. However, training the forecasting tool with raw power measurements might lead to inaccurate results when past production measurements are altered by the adoption of APC strategies and thus the measured power is not the maximum available. Fig. 3 depicts a situation where a PV plant operates in APC regime (star). The production at this working point is not representative of the true irradiance potential, which corresponds instead to the maximum power point (dot). To solve this problem,

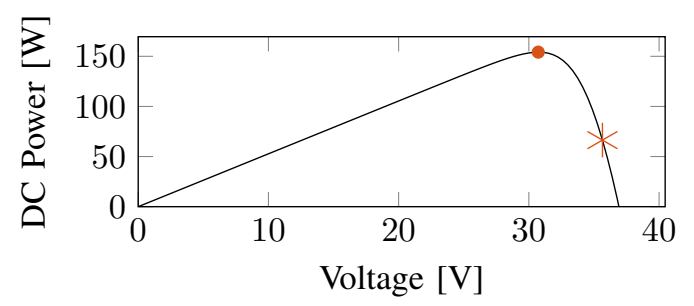

Fig. 3. Example of measured power (star) and maximum power (dot) shown for one PV module.

we first apply a model-based estimator (with parameters $\mathcal{P}_{p}$ ) to reconstruct the DC maximum available power, $\tilde{P}_{d c}$, from measurements of the DC voltage $v$, current $i$, and module temperature $\theta$ (Section III-A1). Then, we use a model of the converter (with parameters $\mathcal{P}_{c}$ ) to estimate the AC maximum power, $\tilde{P}$ (Section III-A2). The procedure is shown in Fig. 4. Historical values of $\tilde{P}$ are then used to train a forecasting tool. The procedure, described in [23], allows to improve the performance of time-series-based forecasting tools compared to the direct forecast, if the system can operate in nonMPPT, by ensuring that the forecasting models are trained with data that are representative of real dynamics of the irradiance/maximum available power. In what follows we detail the overall methodology necessary to predict the PV plant power capability and the related uncertainty. The adopted models, used on-line, are presented in Section III-A, while Section III-B describes how to off-line assess their parameters.

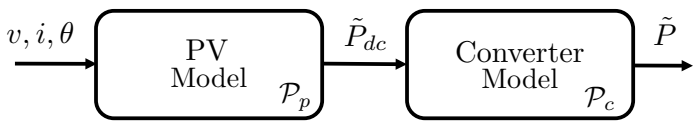

Fig. 4. Maximum Available Power Estimator. The AC maximum power value can be reconstructed using the PV and converter model. The inputs are the measured DC voltage and current $(v, i)$, and the cell temperature $\theta$.

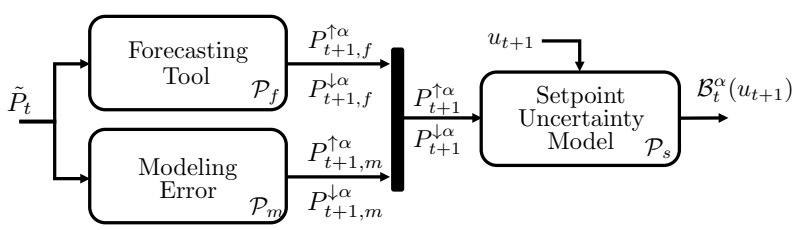

Fig. 5. On-line modelling chain.

\section{A. Models}

The on-line process, depicted in Fig. 5, is as follows. At each time-step $t$, the measurements $v_{t}, i_{t}, \theta_{t}$ are used as inputs to the maximum available power estimator in Fig. 4 to get $\tilde{P}_{t}$. This value is used as input to i) a forecasting tool (with parameters $\mathcal{P}_{f}$ ) to deliver the upper and lower bounds of the one-step-ahead prediction interval $\left(P_{t+1, f}^{\uparrow \alpha}, P_{t+1, f}^{\downarrow \alpha}\right)$ and ii) a modeling error block (with parameters $\mathcal{P}_{m}$ ) to compute the upper and lower bounds accounting for the PV and converter model uncertainty $\left(P_{t+1, m}^{\uparrow \alpha}, P_{t+1, m}^{\downarrow \alpha}\right)$. A conservative approach to compute the global uncertainty associated to the one-stepahead prediction is to consider the extreme bounds between the model bounds and the forecasting intervals ${ }^{3}$ :

$$
\begin{aligned}
& P_{t+1}^{\uparrow \alpha}=\max \left(P_{t+1, f}^{\uparrow \alpha}, P_{t+1, m}^{\uparrow \alpha}\right) \\
& P_{t+1}^{\downarrow \alpha}=\min \left(P_{t+1, f}^{\downarrow \alpha}, P_{t+1, m}^{\downarrow \alpha}\right) .
\end{aligned}
$$

These global bounds $\left(P_{t+1}^{\uparrow \alpha}, P_{t+1}^{\downarrow \alpha}\right)$ are then used as an input for a block that models the ability of the power converter to track a power setpoint (with parameters $\mathcal{P}_{s}$ ). This block outputs a set-valued function, $\mathcal{B}_{t}^{\alpha}\left(u_{t+1}\right)$, which represents the overall uncertainty of tracking a power setpoint $u_{t+1}$. Thus, $\mathcal{B}_{t}^{\alpha}\left(u_{t+1}\right)$ include all the uncertainties related to the PV plant future operation. We next describe the different blocks involved in the methodology: PV model, converter model, forecasting tool, modeling error, and setpoint uncertainty model. We discuss the off-line assessment of the models parameters $\mathcal{P}$ in Section III-B.

1) PV Model: Equivalent circuit models are usually adopted to represent the electrical output of a PV cell, [26]. We select here the five-parameter model proposed in [27], extended to an entire PV array. This is the most used model for PV simulation, thanks to its simplicity and accuracy, since it only needs information from the PV module datasheet. The model for a single cell is shown in Fig. 6. We denote $R_{s}, R_{p}$ the series and shunt resistances and $I_{p}, i_{D}$ the light and saturation currents respectively. We indicate with * the irradiance and temperature at Standard Test Conditions (STC) ${ }^{4}$. We solve the inversed diode equation to estimate the irradiance

\footnotetext{
${ }^{3} \mathrm{~A}$ detailed analysis of these uncertainties is reported in Section IV-B.

${ }^{4}$ Temperature $\theta^{*}=25^{\circ} \mathrm{C}$ and irradiance $G^{*}=1000 \mathrm{~W} \mathrm{~m}^{-2}$.
} 


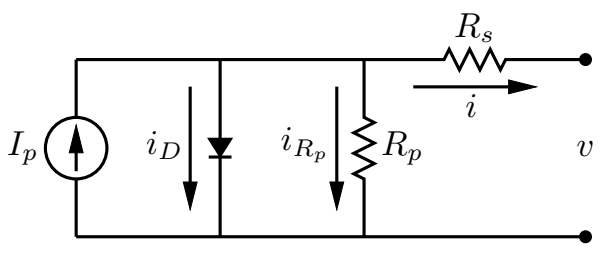

Fig. 6. The five-parameter circuital model of a PV cell, [27]

$$
\begin{aligned}
& \tilde{G}_{t}\left(v_{t}, i_{t}, \theta_{t}\right):^{5} \\
& \tilde{G}_{t}=\frac{i_{t}+i_{D} n_{p}\left(\theta_{t} / \theta^{*}\right)^{3} \exp \left(E_{g}^{*} / k \theta^{*}-E_{g t} / k \theta_{t}\right)\left[\exp \left(q \frac{v_{t}+R_{s} i_{t}\left(n_{s} / n_{p}\right)}{n_{r} k \theta_{t} n_{s}}\right)-1\right]}{\frac{1}{G^{*}}\left[n_{p}\left(I_{p}+\alpha_{I_{S C}}\left(\theta_{t}-\theta^{*}\right)\right)-\frac{v_{t}+R_{s} i_{t}\left(n_{s} / n_{p}\right)}{R_{p}\left(n_{s} / n_{p}\right)}\right]},
\end{aligned}
$$

where $n_{p}$ and $n_{s}$ are the number of cells in parallel and series in a PV module, $\alpha_{I_{S C}}$ the short circuit current temperature coefficient, $k$ the Boltzmann constant, $q$ the electron charge, $E_{g}^{*}$ the material band-gap energy at $\theta^{*}$, and $n_{r}$ the diode ideality factor. $E_{g t}$ is calculated from $E_{g}^{*}$ and $\theta_{t}$ as described in [27]. The parameters of the module model are thus $\mathcal{P}_{p}=\left\{R_{s}, R_{p}, I_{p}, i_{D}, n_{r}, n_{s}, n_{p}, E_{g}^{*}, \alpha_{I_{S C}}\right\}$. The estimated irradiance $\tilde{G}_{t}$ is then used, together with the cell temperature $\theta_{t}$, to compute the maximum DC power. In particular, solving the five-parameter model allows to obtain the $i$ - $v$ curve of the PV array at the conditions defined by the couple $(\tilde{G}, \theta)$. The DC maximum power is simply calculated as the maximum of the product of the $i-v$ curve coordinates. The real-time calculation of the DC maximum power takes less than $15 \mathrm{~ms}$ on a personal computer (Intel Core i7-6600 2.6 GHz, with a 12 GB RAM). This is a key aspect if considering the high reporting rate of predictions for microgrid applications.

2) Converter Model: The converter model allows computing the AC power from the DC power and the DC voltage. Two main models are available in the literature, [29] and [30]. Both require fitting the parameters using datasheet and operational data. In our case, fitting a simple quadratic model led to a better accuracy:

$$
\tilde{P}_{t}=\beta_{0}+\beta_{1} \tilde{P}_{d c, t}+\beta_{2} v_{t}+\beta_{11} \tilde{P}_{d c, t}^{2}+\beta_{12} \tilde{P}_{d c, t} v_{t}+\beta_{22} v_{t}^{2},
$$

where $\tilde{P}_{t}$ is the AC power (response variable), $\tilde{P}_{d c, t}$ the DC power, $v_{t}$ the DC voltage (the predictors), and $\mathcal{P}_{c}=$ $\left\{\beta_{0}, \beta_{1}, \beta_{2}, \beta_{11}, \beta_{12}, \beta_{22}\right\}$ are the model coefficients.

3) Forecasting Tool: To compute short-term prediction intervals (PIs), we adopt the method proposed in [31] directly applied to the $\mathrm{AC}$ power. It consists in clustering historical differentiated maximum power estimations using the k-means clustering algorithm, [32]. The clustering, performed off-line, is based on two influential variables: i) the average power and ii) the power variability, calculated on a rolling time window considering the last three AC maximum power estimations. At each time-step, when a new AC maximum power estimation $\tilde{P}$ is available, the influential variables are computed on-line. The Euclidean distance between the centroids and the actual influential variables is then used as the similarity criterion to identify the representative cluster. Then, we use the upper

\footnotetext{
${ }^{5}$ Refer to [23], [28] to see how Eq. (3) is inferred.
}

and lower quantiles, $\mathcal{P}_{f}=\left\{q_{f}^{\uparrow \alpha}, q_{f}^{\downarrow \alpha}\right\}$, extracted from the representative cluster, to calculate the prediction bounds, at a given confidence level $\alpha$. In particular, we sum the quantiles to the estimated $\mathrm{AC}$ maximum power:

$$
\begin{aligned}
& P_{t+1, f}^{\uparrow \alpha}=\tilde{P}_{t}+q_{f}^{\uparrow \alpha}, \\
& P_{t+1, f}^{\downarrow \alpha}=\tilde{P}_{t}+q_{f}^{\downarrow \alpha} .
\end{aligned}
$$

It is worth noting that the k-means clustering of the training is computed off-line on historical data (see Section III-B) and the on-line computation of the PI takes less than $0.5 \mathrm{~ms}$ on a personal computer.

4) Modeling Error: The model-based strategy to reconstruct $\tilde{P}$ ensure that the forecasting tool learns from training data that are representative of the maximum power, even when the system is not operating in MPPT mode. However, the obtained prediction interval does not account for the error associated to the PV/converter models, needed in the chain to reconstruct the maximum available power. For this reason, a set of AC measurements, $\{\bar{V}, \bar{I}\}$, see Fig. 2, is used to account for the modeling error defined at each time step as:

$$
e_{t}=\tilde{P}_{t}-P_{t}\left(\bar{V}_{t}, \bar{I}_{t}\right),
$$

where $\tilde{P}_{t}$ is the AC maximum power estimation and $P_{t}$ the ground truth value, computed from $\left(\bar{V}_{t}, \bar{I}_{t}\right)$. This error captures all what is not captured by the forecasting model. We consider the modeling error distribution on a training set, and we extract the upper and lower quantiles of the set, $\mathcal{P}_{m}=\left\{q_{m}^{\uparrow \alpha}, q_{m}^{\downarrow \alpha}\right\}$, corresponding to the target confidence level $\alpha$. The uncertainty bounds associated to the model are defined as:

$$
\begin{aligned}
& P_{t+1, m}^{\uparrow \alpha}=\tilde{P}_{t}+q_{m}^{\uparrow \alpha}, \\
& P_{t+1, m}^{\downarrow \alpha}=\tilde{P}_{t}+q_{m}^{\downarrow \alpha} .
\end{aligned}
$$

5) Setpoint Uncertainty Model: The uncertainty on tracking a power setpoint depends on the internal control law of the converter. In particular, for a given setpoint $u$, we have a set of possible $\mathrm{AC}$ active and reactive power injections $(P, Q)$, for which we can compute the distances $d P(u)=P^{*}-P(u)$ and $d Q(u)=Q^{*}-Q(u)$. We define $\left(\delta_{P}^{\uparrow \alpha}(u), \delta_{P}^{\downarrow \alpha}(u)\right)$ and $\left(\delta_{Q}^{\uparrow \alpha}(u), \delta_{Q}^{\downarrow \alpha}(u)\right)$ as the upper and lower quantiles extracted from the $d P(u)$ and $d Q(u)$ distributions, respectively. For a given setpoint $u_{t}=\left(P_{t}^{*}, Q_{t}^{*}\right)$, the implemented power lays in a rectangular set with a certain confidence level $\alpha$, i.e. $y_{t} \in \mathcal{R}^{\alpha}\left(u_{t}\right)$ where:

$$
\begin{aligned}
\mathcal{R}^{\alpha}\left(u_{t}\right)= & \left\{(P, Q) \in \mathbb{R}^{2} \mid\right. \\
& \delta_{P}^{\downarrow \alpha}\left(u_{t}\right) \leq P_{t}^{*}-P \leq \delta_{P}^{\uparrow \alpha}\left(u_{t}\right), \\
& \left.\delta_{Q}^{\downarrow \alpha}\left(u_{t}\right) \leq Q_{t}^{*}-Q \leq \delta_{Q}^{\uparrow \alpha}\left(u_{t}\right)\right\},
\end{aligned}
$$

$\mathcal{P}_{s}=\left\{\delta_{P}^{\downarrow \alpha}, \delta_{P}^{\uparrow \alpha}, \delta_{Q}^{\downarrow \alpha}, \delta_{Q}^{\uparrow \alpha}\right\}$ contains all the extracted quantiles computed off-line for different values of $u$.

The sets of parameters $\mathcal{P}_{p}, \mathcal{P}_{c}, \mathcal{P}_{f}, \mathcal{P}_{m}, \mathcal{P}_{s}$, are computed during an off-line phase, as described in the following. 


\section{B. Models Parameters: Off-line Assessment}

The parameters of the module model $\mathcal{P}_{p}$ can be computed off-line using the procedure in [33] that only needs the module datasheet as input information. The converter model coefficients defining $\mathcal{P}_{c}$ are obtained by fitting a regression model on a set of data obtained by operating the converter from zero to the maximum rated power. To train the forecasting tool (and compute $\mathcal{P}_{f}$ ) we consider a set of $N$ historical measurements $\{v, i, \theta\}$ and use the maximum available power estimator, in Section III-A1, to get $\tilde{P}$ for each set of measurements. The obtained $N$ historical estimations of $\tilde{P}$ are used to build the clusters of the selected probabilistic forecasting tool, see Section III-A3. The clustered data are used to extract the quantiles that define $\mathcal{P}_{f}$. The quantiles of the modeling error distribution are used to define $\mathcal{P}_{m}$. In practice, for period of length $M$, we let the PV converter work in MPPT, and thus the maximum available power can be directly measured. The training set used to define the error should cover different irradiance and temperature conditions, for example by selecting few entire days characterized by different weather conditions. Finally, the setpoint uncertainty is assessed by requesting specific power setpoints to the converter, by sweeping the whole $P Q$ capability set. We then extract the quantiles from the distributions of the observed distances $(d P(u), d Q(u))$, for all the requested setpoints $u$. This allows to define $\mathcal{P}_{s}$.

\section{RESULTS}

\section{A. PV and Converter Models Performance}

In the case of the PV model, the method can reconstruct the theoretical maximum power output with a normalized root mean squared error (nRMSE) of $0.5-6 \%$, see [23]. For the converter model, the values of the coefficients from Eq. (4) are shown in Table I. Fig. 7 compares the estimations from

TABLE I

CONVERTER MOdel COEFFicients

\begin{tabular}{cccccc}
$\beta_{0}$ & $\beta_{1}$ & $\beta_{2}$ & $\beta_{11}$ & $\beta_{12}$ & $\beta_{22}$ \\
\hline-220 & 0.92 & -0.60 & $-2.89 \cdot 10^{-6}$ & $2.06 \cdot 10^{-5}$ & -0.01 \\
\hline
\end{tabular}

the fitting to the measured $\mathrm{AC}$ power values for an entire day of measurements, presenting an error of $1.6 \%$ (nRMSE).

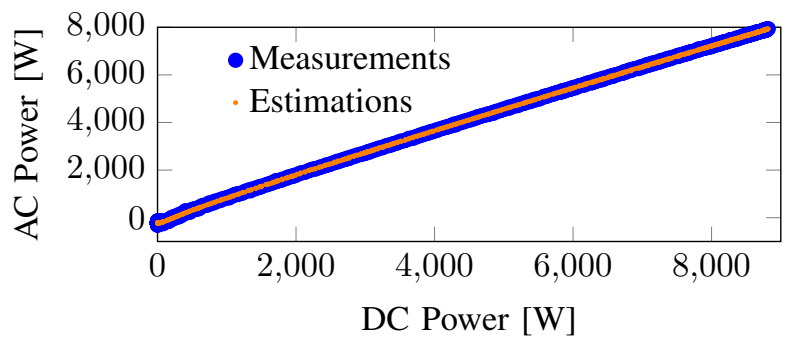

Fig. 7. The AC power, estimated using the converter model described in Section III-A2, is compared with the measured AC power.

\section{B. Maximum Available Power Uncertainty Assessment}

In this section, we quantify the prediction uncertainty of the AC maximum power considering different forecast horizons (100 ms, $1 \mathrm{~s}, 1 \mathrm{~min}$ and $5 \mathrm{~min}$ ), and different levels of curtailed energy $(0 \%, 2 \%$ and $30 \%)$. The latter is computed as the percentage of curtailed energy over the maximum available in the testing period. The metrics are adapted from [34]. The first metric is the bound Coverage Probability (CP) which counts the number of times the realization falls inside the PI for a given confidence level $\alpha$ :

$$
\mathrm{CP}=\frac{1}{L} \sum_{t=1}^{L} c_{t}, \quad c_{t}= \begin{cases}1, & P_{t+1 \mid t}^{\downarrow \alpha} \leq P_{t+1} \leq P_{t+1 \mid t}^{\uparrow \alpha} \\ 0, & \text { otherwise }\end{cases}
$$

where $L$ is the total number testing instances.

Then, the interval normalized Averaged Width (AW):

$$
\mathrm{AW}=\frac{1}{L P_{\max }} \sum_{t=1}^{L}\left(P_{t+1 \mid t}^{\uparrow \alpha}-P_{t+1 \mid t}^{\downarrow \alpha}\right)
$$

where $P_{\max }=13 \mathrm{~kW}$ is the rated power of the converter. Bounds are considered accurate when $\mathrm{CP} \geq \alpha$ and the AW is low. The bounds are computed: i) as in Eqs. (1)-(2), i.e., considering the worst case between modeling and forecast uncertainty (Global), ii) considering only the forecast uncertainty (Forecast), and iii) considering only the modeling uncertainty (Model). Results are summarized in Table II and Table III for a target confidence level of $95 \%$. The length of the training set and the number of clusters for the k-mean-based algorithm are selected accordingly to [31]. In particular, we consider a training set of 4 days for the $100 \mathrm{~ms}$ and $1 \mathrm{~s}$ horizons, and of 10 days for the 1 and 5 min horizons. The testing set is of 2 days. The number of clusters is $100,25,15$, and 5 for $100 \mathrm{~ms}, 1 \mathrm{~s}, 1 \mathrm{~min}$, and $5 \mathrm{~min}$, respectively. In our analysis, the same training set is used to extract the quantiles for the modeling error. From Table II we observe that only the global prediction chain is able to deliver bounds with high coverage ( $\mathrm{CP}$ is similar to the target confidence level). At short-term horizons (below the minute scale), the forecast uncertainty is low (Table III), and accounting for the model uncertainty is necessary to guarantee a decent coverage (higher than $85 \%$ ). At horizons above the minute scale, the forecast uncertainty is instead dominant. As example, Fig. 8 shows forecast and model bounds for $1 \mathrm{~s}$ and $1 \mathrm{~min}$ forecast horizons, see Fig. $8 \mathrm{a}$ and $8 \mathrm{~b}$, respectively. It is possible to see that at $1 \mathrm{~s}$ horizon the model bounds are of bigger magnitude and are necessary to guarantee the target coverage probability. On the contrary, above $1 \mathrm{~min}$ horizon the forecast uncertainty is higher, and it has the highest contribution to the global coverage. From Table II we can see that the global CP is lower than $\alpha$ only at low forecast horizon (second and sub-second) with curtailment, highlighted with underlines. This is due to the fact that the selected maximum power estimator (based on measurements of the PV system) is very sensitive to the operating conditions, and a higher modeling error is expected when the system is far from MPPT conditions. However, in common plants, the modeling error can be computed only with respect to a ground truth value obtained by leaving the system in MPPT and comparing the measured and estimated 
TABLE II

COVERAGe Probability-CP [\%], FOR $\alpha=95 \%$. RESUltS ARE SHOWN FROM 100 MS TO 5 MIN FORECAST HORIZON. UNDERLINES REFER TO GLOBAL CP LOWER THAN $\alpha$.

\begin{tabular}{|c|c|c|c|}
\cline { 2 - 4 } \multicolumn{1}{c|}{} & \multicolumn{3}{c|}{ Global Uncertainty } \\
\hline Forecast Horizon & MPPT & $\mathbf{2 \%}$ Curt. & 30\% Curt. \\
\hline $100 \mathrm{~ms}$ & 98.1 & $\underline{93.2}$ & $\underline{86.0}$ \\
\hline $1 \mathrm{~s}$ & 97.6 & $\underline{93.0}$ & $\underline{86.1}$ \\
\hline $1 \mathrm{~min}$ & $98.0-8.7$ & 97.0 & 96.7 \\
\hline $5 \mathrm{~min}$ & $98.0-15$ & 95.7 & 96.5 \\
\hline
\end{tabular}

\begin{tabular}{|c|c|c|}
\hline \multicolumn{3}{|c|}{ Forecast Uncertainty } \\
\hline MPPT & 2\% Curt. & 30\% Curt. \\
\hline $22.7-0.27$ & 31 & 27 \\
\hline $28.8-0.3$ & 32.0 & 28 \\
\hline 84.8 & 76.9 & 81.0 \\
\hline 92.6 & 92.0 & 94.0 \\
\hline
\end{tabular}

\begin{tabular}{|c|c|c|}
\hline \multicolumn{3}{|c|}{ Model Uncertainty } \\
\hline MPPT & 2\% Curt. & 30\% Curt. \\
\hline 97.4 & 89 & 84 \\
\hline 96.8 & 87 & 83 \\
\hline 79.9 & 69.8 & 53 \\
\hline 58 & 40.6 & 38.0 \\
\hline
\end{tabular}

TABLE III

NORMALIZED AVERAGE WIDTH-AW [\%], FOR $\alpha=95 \%$. RESULTS ARE SHOWN FROM 100 MS TO 5 MIN FORECAST HORIZON.

\begin{tabular}{|c|c|c|c|}
\cline { 2 - 4 } \multicolumn{1}{c|}{} & \multicolumn{3}{c|}{ Global Uncertainty } \\
\hline Forecast Horizon & MPPT & $\mathbf{2 \%}$ Curt. & 30\% Curt. \\
\hline $100 \mathrm{~ms}$ & 2.28 & 3.4 & 2.6 \\
\hline $1 \mathrm{~s}$ & 2.1 & 3.2 & 2.5 \\
\hline $1 \mathrm{~min}$ & 8.7 & 14.9 & 12 \\
\hline $5 \mathrm{~min}$ & 15 & 25.4 & 20 \\
\hline
\end{tabular}

\begin{tabular}{|c|c|c|}
\hline \multicolumn{3}{|c|}{ Forecast Uncertainty } \\
\hline MPPT & 2\% Curt. & 30\% Curt. \\
\hline 0.27 & 0.2 & 0.78 \\
\hline 0.3 & 0.3 & 0.75 \\
\hline 6.7 & 11.9 & 9.4 \\
\hline 15 & 24.1 & 20 \\
\hline
\end{tabular}

\begin{tabular}{|c|c|c|}
\hline \multicolumn{3}{|c|}{ Model Uncertainty } \\
\hline MPPT & 2\% Curt. & 30\% Curt. \\
\hline 2.15 & 2.2 & 2.2 \\
\hline 2.15 & 2.2 & 2.2 \\
\hline 2.15 & 2.2 & 2.2 \\
\hline 2.15 & 2.2 & 2.2 \\
\hline
\end{tabular}

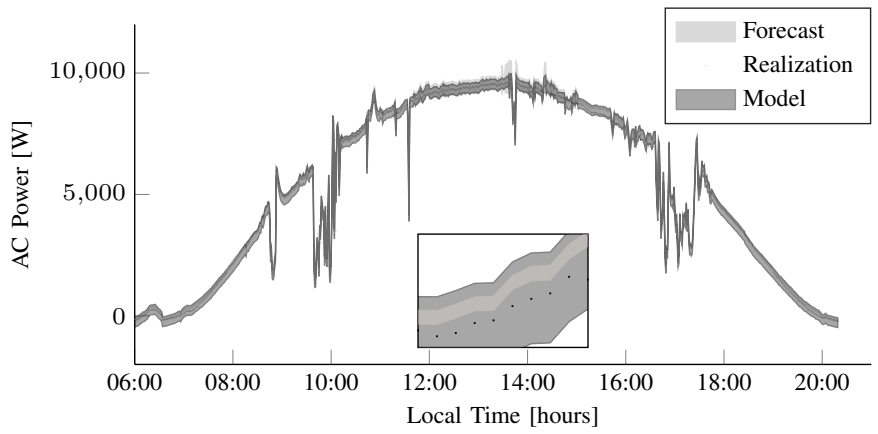

(a) Prediction Uncertainty, $1 \mathrm{~s}$ forecast horizon

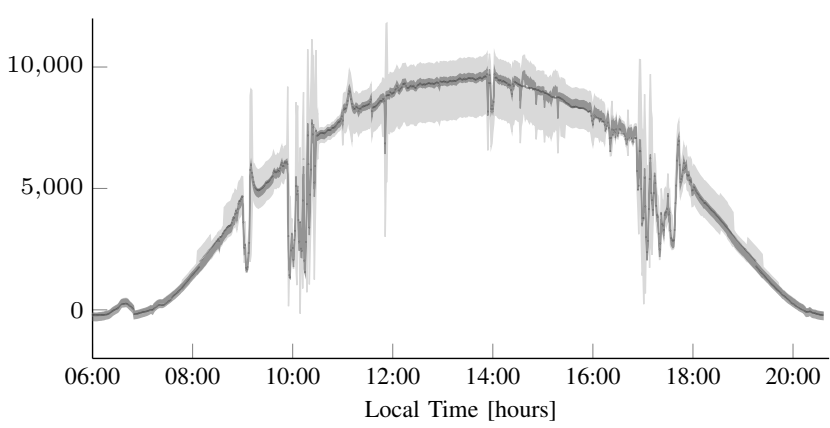

(b) Prediction Uncertainty, 1 min forecast horizon

Fig. 8. Comparison of the forecast bounds, model bounds, and realizations. One day of active power generation is shown for the experimental setup described in Section II.

power. Thus, the error defined in Eq. (7) only accounts for MPPT and not for curtailed conditions. This explains the lower coverage of the bounds when curtailment actions are adopted. Furthermore, this higher modeling error affects the global CP only at low horizons (lower than the minute scale), i.e. when accounting for the modeling error is more relevant. To understand the higher error associated with the DC maximum power estimation when far from MPPT, we perform a sensitivity analysis with respect to the model's parameters. In particular, we change one-factor-at-a-time (OAT approach), [35]. Fig. 9 shows the sensitivity of the DC maximum power estimation $\left(\tilde{P}_{d c}\right)$ with respect to the parameters $i_{D}, n_{r}$, and $R_{s}$. We recall from Section III-A1 that, these parameters, together with $R_{p}$ and $I_{p}$, are computed from datasheet parameters at STC and then updated to account for their dependency of the temperature and irradiance. The sensitivity is done considering two values of irradiance (500 and $\left.800 \mathrm{~W} / \mathrm{m}^{2}\right)$ and constant temperature. For this assessment we consider that the initial operating point of the PV system can be MPPT, open circuit (OC), and curtailed (CUR, for this case we select a point halfway between MPPT and OC). We observe that when operating far from MPPT, the estimation is very sensitive to the values of $i_{D}$ and $n_{r}$. For example, an error on the estimation of $n_{r}$ of $10 \%$ can lead to an $80 \%$ error on the estimation of the DC maximum power when the system operates close to OC conditions. The value of the series resistance $R_{s}$ similarly affects the MPPT and curtailed conditions, and less affects OC conditions.

We can conclude that when referring to forecast horizons below 1 minute, where the model uncertainty prevails in the definition of the global power prediction uncertainty, it is particularly important to invest in an accurate model of the PV model and precise equations that account for the dependency of the five parameters on the weather conditions.

\section{Setpoint Tracking Uncertainty Assessment}

In this section we quantify the uncertainty produced by the power converter to track external setpoints. We recall from Sections III-A5 and III-B that for a given request $u=\left(P^{*}, Q^{*}\right)$, we have a set of possible injections $(P, Q)$, and a corresponding set of possible distances $d P(u)=$ $P^{*}-P(u)$ and $d Q(u)=Q^{*}-Q(u)$. In order to assess the uncertainty associated with the setpoint implementation, we perform several tests by sending to the converter different requests $u$, sweeping the whole $P Q$ admissible set, with a step size of $100 \mathrm{~W} / 100 \mathrm{VAr}$, for the active and reactive power 


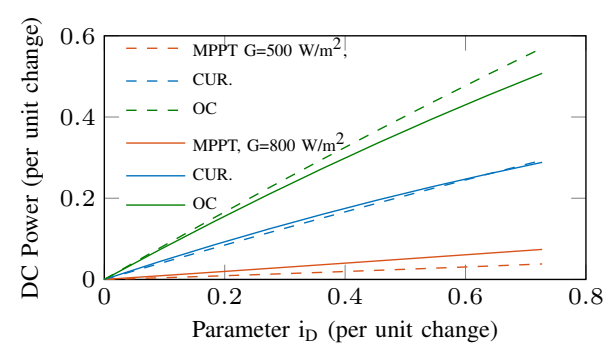

(a) Sensitivity on $i_{D}$

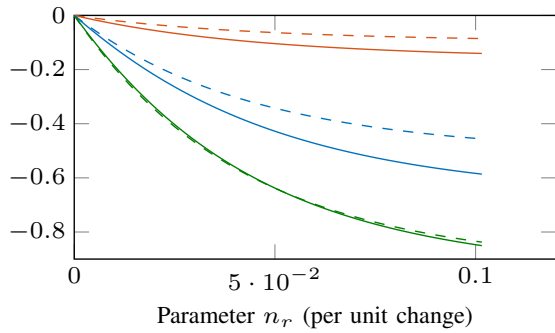

(b) Sensitivity on $n_{r}$

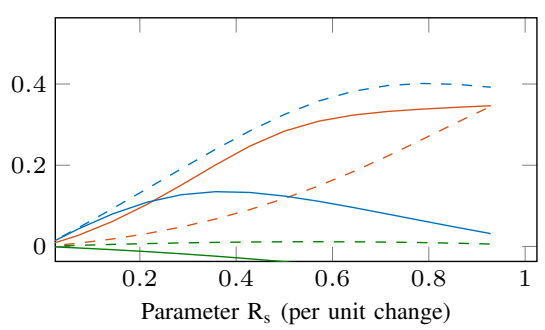

(c) Sensitivity on $R_{s}$

Fig. 9. Per unit change of the DC maximum power estimation with respect to the per unit change of the input parameter. The base unit quantities of the DC maximum power and of the parameters are those originally obtained from the model in [27].

respectively ${ }^{6}$. The quantiles defining $\mathcal{P}_{s}$ are extracted from the $d P(u)$ and $d Q(u)$ distributions recorded during these offline tests. As example, Fig. 10 shows the quantiles $\left(\delta_{P}^{\downarrow}, \delta_{P}^{\uparrow}\right)$ for a confidence level $\alpha=95 \%$ and for different couples $\left(P^{*}, Q^{*}\right)$. Similar results are obtained for $Q$. The quantiles can be stored in a 2D look-up table that returns in real-time the quantiles $\left(\delta_{P}^{\uparrow}, \delta_{P}^{\downarrow}\right)$ and $\left(\delta_{Q}^{\uparrow}, \delta_{Q}^{\downarrow}\right)$ as a function of a given request $\left(P^{*}, Q^{*}\right)$. Fig. 10 shows that this uncertainty can reach magnitudes of $600 \mathrm{~W}$ that corresponds to $\approx 5 \%$ of the plant rated power. Section V illustrates how to formally include this uncertainty in the overall process, and finally define $\mathcal{B}^{\alpha}(u)$.

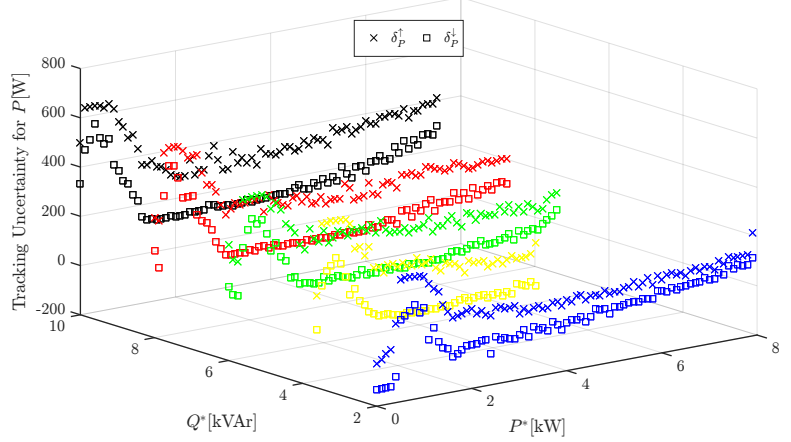

Fig. 10. Setpoints Tracking Uncertainty on Active Power.

\section{Application: PV Agent Design}

We show here how it is possible to combine the discussed models and uncertainties to design an agent for a PV system that is meant to periodically inform a grid controller (GC) about the PV plant capabilities. Recall from Section III that these capabilities may continuously change as a function of the external perturbation $w$ and the converter limitations $e$. Inspired by [36], we define two elements that can represent the way the PV system can be controlled (i) the Power Flexibility $\mathcal{A}$ and (ii) the Controllability Uncertainty $\mathcal{B}$. Note that, the PV Agent looks for expressing the controllability of the PV system at a given time-period in the future, i.e., at the time when it expects to receive a new power setpoint. We assume that, if the GC sends setpoints fast enough, the information generated by the PV Agent can be computed in discrete-time,

\footnotetext{
${ }^{6}$ Each test lasts until the maximum available power is reached, accordingly to given irradiance and temperature conditions.
}

considering that it remains constant between two consecutive time-steps. Therefore, we target to have a message update in the sub-second scale so that we can cope with the fastest dynamics of real PV systems. This serves to simplify the information sent to the GC without the need of explicitly including the time dependence of the external perturbations between consecutive time-steps. We next describe how to compute these two elements.

\section{A. Power Flexibility $\mathcal{A}$}

In general, we consider that the power flexibility of a controllable PV system can be defined as a function of (i) the predicted maximum available power $P_{t+1}^{\uparrow \alpha}$, (ii) the converter rated power $S_{r}$, and (iii) a minimum power factor constraint $\mathrm{PF}_{\min }$. This last is typically required to minimize the reactive power flows in the grid and to comply with quality-of-service norms, generally associated to voltage control.

As for the analysis presented in Section III, the maximum available power is defined by the upper bound of the global prediction bound, $P_{t+1}^{\uparrow \alpha}$. Considering this, and $S=P+j Q$ as the complex power, we define $\mathcal{A}$ as the set in the $P Q$-plane where a GC can request a setpoint $u$ in the next time-step, i.e., $u_{t+1} \in \mathcal{A}_{t}$. This is,

$$
\begin{aligned}
\mathcal{A}_{t}= & \left\{(P, Q) \in \mathbb{R}^{2} \mid\right. \\
& \left.0 \leq P \leq P_{t+1}^{\uparrow \alpha}, \frac{|P|}{\|S\|} \geq \mathrm{PF}_{\min },\|S\| \leq S_{r}\right\}
\end{aligned}
$$

The graphical representation of $\mathcal{A}$ is shown in Fig. 11 .

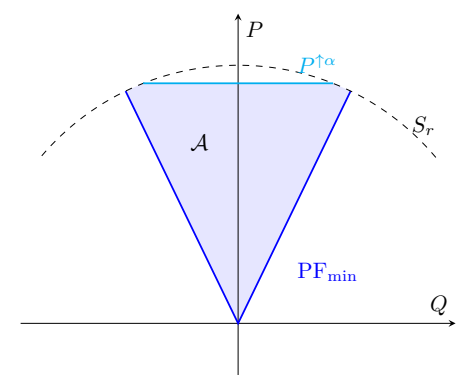

Fig. 11. PV system Power Flexibility. 


\section{B. Controllability Uncertainty $\mathcal{B}$}

As previously shown in Section III, the uncertainty of implementing a given setpoint is strongly dependent on two factors (i) the maximum available $\mathrm{AC}$ power (from forecast and model) and (ii) the setpoint tracking accuracy. Let us first focus on the former. The set defined by the uncertainty of the maximum available power, is a segment that depends on $\mathrm{PF}_{\text {min }}$. The definition for any $u_{t+1}=\left(P_{t+1}^{*}, Q_{t+1}^{*}\right) \in \mathcal{A}_{t}$ is in Eq. (14). $P_{t+1}^{\prime}$ and $Q_{t+1}^{\prime}$ are expressed as:

$$
\begin{array}{r}
P_{t+1}^{\prime}=Q_{t+1}^{*} \tan \left(\arccos \left(\mathrm{PF}_{\min }\right)\right), \\
Q_{t+1}^{\prime}=\operatorname{sign}\left(Q_{t+1}^{*}\right) P_{t+1}^{\downarrow \alpha} \frac{\sqrt{1-\mathrm{PF}_{\min }^{2}}}{\mathrm{PF}_{\min }} .
\end{array}
$$

The graphical representation is shown in Fig. 12a.

Now, as presented in Section III-A5, the uncertainty of the power converter to deploy a power setpoint is defined by Eq. (10). The set defined by this uncertainty for any $\left(P_{t+1}^{*}, Q_{t+1}^{*}\right) \in \mathcal{A}_{t}$, shown in Fig. 12b, is thus:

$$
\mathcal{B}_{2, t}^{\alpha}\left(u_{t+1}\right)=\mathcal{R}^{\alpha}\left(u_{t+1}\right) .
$$

The overall uncertainty on deploying a power setpoint $u_{t+1}$ can be, therefore, written as follows (see Fig. 12c):

$$
\mathcal{B}_{t}^{\alpha}\left(u_{t+1}\right)=\bigcup_{(p, q) \in \mathcal{B}_{1, t}^{\alpha}\left(u_{t+1}\right)} \mathcal{B}_{2, t}^{\alpha}\left(u_{t+1}\right) .
$$

The two quantities $\mathcal{A}, \mathcal{B}^{\alpha}$ can be sent to the GC upon request. Namely, upon reception of a setpoint $u_{t}$, the agent will compute $\mathcal{X}_{t}=\left(\mathcal{A}_{t}, \mathcal{B}_{t}^{\alpha}\right)$ that represents how the PV system will be able to implement a setpoint at instant $t+\Delta t$. Since the proposed tool is capable of computing $\mathcal{B}^{\alpha}$ for different forecast horizons, the value of $\Delta t$ can be either a defined apriori or in real-time along with the setpoint. In practice, the GC and the PV Agent should agree on a message format for both $u$ and $\mathcal{X}$, so that the communication burden is minimized. For instance, if the GC knows beforehand the shape of sets $\mathcal{A}$ and $\mathcal{R}$, the PV Agent could only send $\left(P_{t+1}^{\uparrow \alpha}, S_{r}, \mathrm{PF}_{\min }\right)$ for $\mathcal{A}$ and the parameters of the rectangle that defines the setpoint tracking uncertainty, $\mathcal{R}^{\alpha}(u), \mathcal{P}_{s}=\left\{\delta_{P}^{\downarrow \alpha}, \delta_{P}^{\uparrow \alpha}, \delta_{Q}^{\downarrow \alpha}, \delta_{Q}^{\uparrow \alpha}\right\}$ (for $u \in \mathcal{A}$ ) to represent $\mathcal{B}^{\alpha}$.

\section{DISCUSSION}

\section{A. Extension to Energy Dispatch}

In this paper we have focused on the uncertainty assessment of the one-step-ahead power production of a PV plant, including the implementation uncertainty produced by the setpoint tracking algorithm of the PV converter. However, a similar strategy can be used for longer-term problems. For example, in an energy dispatch problem (e.g. [37], [38]), the proposed modelling chain can provide a predefined coverage interval that represents the expected uncertainties of the injected power for multiple time steps in a given time horizon, together with the explicit uncertainties for a given power setpoints.

\section{B. Deployment of the Method in a Real-World Scenario}

In this section, we describe the steps to be undertaken by a modeller interested in implementing the proposed methodology, independently of the location and the weather conditions of the PV plant.

- The PV module datasheet is used to compute the value of the 5 parameters of the single-diode model, as in [33].

- Few days of locally collected measurements of the DC voltage, current, and cell temperature are needed to train the forecasting algorithm and the modelling errors model. However, a periodical retraining is advisable to account for seasonal dynamics. Alternatively, a clear-sky model for the power can be introduced to remove seasonal and daily deterministic dynamics, as proposed in [39].

- Measurements of the DC voltage, DC current, and AC active power are needed to assess off-line the converter efficiency and the setpoint uncertainty. The experiment should sweep the whole $P Q$ capability set of the converter. We note that this information might also be provided by the manufacturer of the converter.

- On-line measurements of the DC voltage, current, and cell temperature are needed to reconstruct the maximum available power, that is then used as input for the forecasting and modelling error models (previously built off-line).

\section{CONCLUSION}

Due to the inherent short-term variability of solar irradiance, predictive control coupled with accurate stochastic models is considered as a suitable way to achieve the controllability of PV generation. This paper tackles the problem of assessing the uncertainty associated with the operation of a controllable PV facility. The analysis included two fundamental aspects: determining the PV generation potential for the next time interval (allowing to identify a region inside the capability curve of the power converter from where it is possible to pick a power setpoint), and characterizing the uncertainty associated to tracking a setpoint inside that region. The former element is determined by using a short-term forecasting method, whereas the latter depends on the converter internal dynamics and is evaluated with a data-driven approach. The proposed grey-box modelling relies on physical models as well as on measurements of the PV currents, voltages, and the cell temperature, all information normally available to the PV plant operator, without the use of extra sensors (e.g., pyranometers). The proposed method was tested considering different forecasting horizons, from $100 \mathrm{~ms}$ to 5 minutes ahead. Experimental results showed that, when considering forecasting horizons below 1 minute, accurate physical models are the key to achieve reliable coverage of the prediction interval. On the other hand, for forecasting horizons larger than 1 minute, the quality of the predictions is mostly affected by the uncertainty associated with the forecasting, which becomes dominant with respect to models performance. As an application, we have shown how the proposed model can be integrated into a predictive control logic for a PV power plant, which can be used to control PV generation in combination with other distributed energy resources. 


$$
\mathcal{B}_{1, t}^{\alpha}\left(u_{t+1}\right)= \begin{cases}\left\{\left(P_{t+1}^{*}, Q_{t+1}^{*}\right)\right\} & 0 \leq P_{t+1}^{*} \leq P_{t+1}^{\downarrow \alpha}, \\ {\left[\left(P_{t+1}^{*}, Q_{t+1}^{*}\right),\left(P_{t+1}^{\prime}, Q_{t+1}^{*}\right)\right] \cup\left[\left(P_{t+1}^{\prime}, Q_{t+1}^{*}\right),\left(P_{t+1}^{\downarrow \alpha}, Q_{t+1}^{\prime}\right)\right]} & P_{t+1}^{\downarrow \alpha} \leq P_{t+1}^{*} \leq P_{t+1}^{\uparrow \alpha},\end{cases}
$$

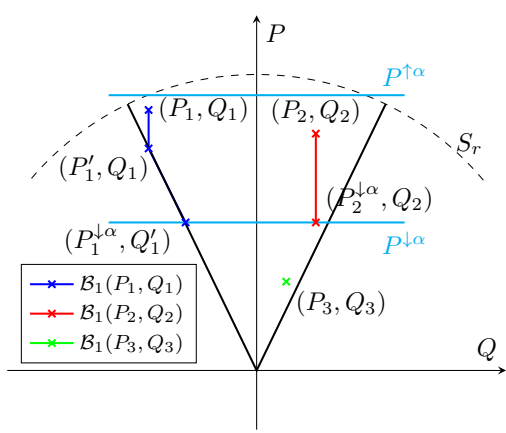

(a) Maximum available power

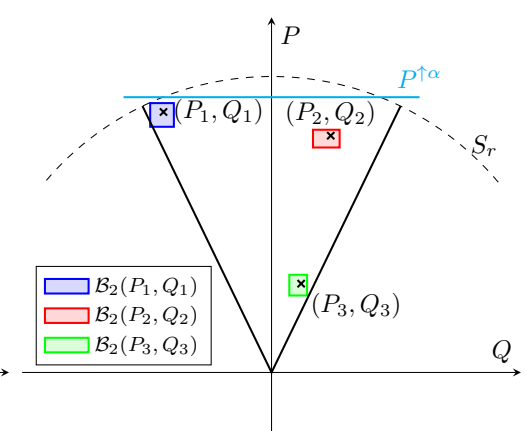

(b) Setpoint tracking accuracy

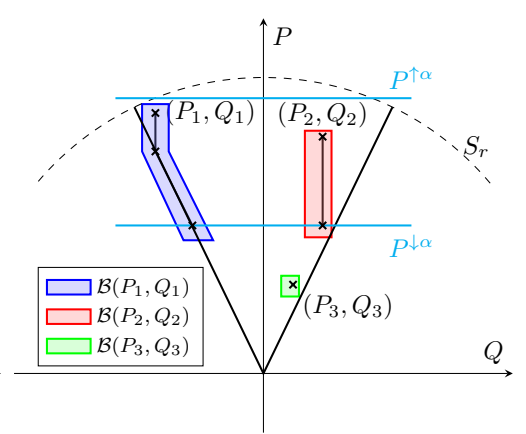

(c) Overall uncertainty

Fig. 12. Examples of Controllability Uncertainty. The subscripts represent three different possible setpoints.

\section{REFERENCES}

[1] D. E. Olivares, A. Mehrizi-Sani, A. H. Etemadi, C. Canizares, R. Iravani, M. Kazerani, A. H. Hajimiragha, O. Gomis-Bellmunt, M. Saeedifard, R. Palma-Behnke et al., "Trends in microgrid control," Smart Grid, IEEE Transactions on, vol. 5, no. 4, pp. 1905-1919, 2014.

[2] J. P. Lopes, N. Hatziargyriou, J. Mutale, P. Djapic, and N. Jenkins, "Integrating distributed generation into electric power systems: A review of drivers, challenges and opportunities," Electric Power Systems Research.

[3] M. H. Bollen and F. Hassan, Integration of distributed generation in the power system. John wiley \& sons, 2011, vol. 80.

[4] CIGRÉ Task Force C6.11, "Development and Operation of Active Distribution Networks,” CIGRÉ, Paris, France, Tech. Rep. 457, 2011.

[5] J. M. Bright, O. Babacan, J. Kleissl, P. G. Taylor, and R. Crook, "A synthetic, spatially decorrelating solar irradiance generator and application to a lv grid model with high pv penetration," Solar Energy, 2017.

[6] G. K. Ari and Y. Baghzouz, "Impact of high pv penetration on voltage regulation in electrical distribution systems," in 2011 International Conference on Clean Electrical Power (ICCEP), June 2011.

[7] E. Demirok, P. C. Gonzalez, K. H. Frederiksen, D. Sera, P. Rodriguez, and R. Teodorescu, "Local reactive power control methods for overvoltage prevention of distributed solar inverters in low-voltage grids," IEEE Journal of Photovoltaics, vol. 1, no. 2, pp. 174-182, 2011.

[8] S. Ghosh, S. Rahman, and M. Pipattanasomporn, "Local distribution voltage control by reactive power injection from pv inverters enhanced with active power curtailment," in PES General Meeting-Conference \& Exposition, 2014 IEEE. IEEE, 2014, pp. 1-5.

[9] E. Dall'Anese, S. V. Dhople, and G. B. Giannakis, "Optimal dispatch of photovoltaic inverters in residential distribution systems," IEEE Transactions on Sustainable Energy, vol. 5, no. 2, pp. 487-497, 2014.

[10] S. Ghosh, S. Rahman, and M. Pipattanasomporn, "Distribution voltage regulation through active power curtailment with pv inverters and solar generation forecasts," IEEE Transactions on Sustainable Energy, 2017.

[11] T. Logenthiran, D. Srinivasan, A. M. Khambadkone, and H. N. Aung, "Multiagent system for real-time operation of a microgrid in real-time digital simulator," IEEE Transactions on Smart Grid, June 2012.

[12] M. Pipattanasomporn, H. Feroze, and S. Rahman, "Multi-agent systems in a distributed smart grid: Design and implementation," in Power Systems Conference and Exposition, 2009. PSCE'09. IEEE/PES. IEEE.

[13] J. M. Guerrero, J. C. Vasquez, J. Matas, L. G. de Vicuna, and M. Castilla, "Hierarchical control of droop-controlled ac and dc microgrids - a general approach toward standardization," IEEE Trans. on Industrial Electronics, vol. 58, no. 1, pp. 158-172, Jan 2011.

[14] X. Lu, K. Sun, J. Guerrero, and L. Huang, "Soc-based dynamic power sharing method with ac-bus voltage restoration for microgrid applications," in IECON 2012 - 38th Annual Conference on IEEE Industrial Electronics Society, Oct 2012, pp. 5677-5682.

[15] A. Vaccaro, V. Loia, G. Formato, P. Wall, and V. Terzija, "A selforganizing architecture for decentralized smart microgrids synchronization, control, and monitoring," IEEE Trans. on Industrial Informatics, vol. 11, no. 1, pp. 289-298, Feb 2015.
[16] H. Liang, B. J. Choi, W. Zhuang, X. Shen, A. S. A. Awad, and A. Abdr, "Multiagent coordination in microgrids via wireless networks," IEEE Wireless Communications, vol. 19, no. 3, pp. 14-22, June 2012.

[17] C. Rehtanz, Autonomous Systems and Intelligent Agents in Power System Control and Operation. Springer, 2003.

[18] J. Antonanzas, N. Osorio, R. Escobar, R. Urraca, F. M. de Pison, and F. Antonanzas-Torres, "Review of photovoltaic power forecasting," Solar Energy, vol. 136, pp. 78 - 111, 2016.

[19] J. J. Roberts, A. A. M. Zevallos, and A. M. Cassula, "Assessment of photovoltaic performance models for system simulation," Renewable and Sustainable Energy Reviews, vol. 72, pp. 1104-1123, 2017.

[20] A. Grantham, Y. R. Gel, and J. Boland, "Nonparametric short-term probabilistic forecasting for solar radiation," Solar Energy, vol. 133, pp. 465-475, 2016.

[21] C. Zogogianni, L. Reyes-Chamorro, M. Paolone, V. Porobic, E. Adzic, and M. Vekic, "Real-time power-reference tracking method for pv converters," in 2017 IEEE Manchester PowerTech, June.

[22] J. Rocabert, A. Luna, F. Blaabjerg, and P. Rodriguez, "Control of power converters in ac microgrids," IEEE Transactions on Power Electronics, vol. 27, no. 11, pp. 4734-4749, Nov 2012.

[23] E. Scolari, F. Sossan, and M. Paolone, "Photovoltaic model-based solar irradiance estimators: Performance comparison and application to maximum power forecasting," IEEE Transactions on Sustainable Energy, vol. PP, no. 99, pp. 1-1, 2017.

[24] H. T. Pedro and C. F. Coimbra, "Assessment of forecasting techniques for solar power production with no exogenous inputs," Solar Energy, vol. 86, no. 7, pp. 2017 - 2028, 2012.

[25] Y.-Z. Li, L. He, and R.-Q. Nie, "Short-term forecast of power generation for grid-connected photovoltaic system based on advanced grey-markov chain," in Energy and Environment Technology, 2009. ICEET'09. International Conference on, vol. 2. IEEE, 2009, pp. 275-278.

[26] V. J. Chin, Z. Salam, and K. Ishaque, "Cell modelling and model parameters estimation techniques for photovoltaic simulator application: A review," Applied Energy, vol. 154, pp. 500 - 519, 2015.

[27] W. D. Soto, S. Klein, and W. Beckman, "Improvement and validation of a model for photovoltaic array performance," Solar Energy, vol. 80, no. 1 , pp. $78-88,2006$.

[28] A. Laudani, F. R. Fulginei, A. Salvini, M. Carrasco, and F. MancillaDavid, "A fast and effective procedure for sensing solar irradiance in photovoltaic arrays," in Environment and Electrical Engineering (EEEIC), 2016 IEEE 16th International Conference on. IEEE, 2016.

[29] W. E. Boyson, G. M. Galbraith, D. L. King, and S. Gonzalez, "Performance model for grid-connected photovoltaic inverters." Sandia National Laboratories, Tech. Rep., 2007.

[30] A. Driesse, P. Jain, and S. Harrison, "Beyond the curves: Modeling the electrical efficiency of photovoltaic inverters," in 2008 33rd IEEE Photovoltaic Specialists Conference, May 2008, pp. 1-6.

[31] E. Scolari, F. Sossan, and M. Paolone, "Irradiance prediction intervals for PV stochastic generation in microgrid applications," Solar Energy, 2016. 
[32] S. P. Lloyd, "Least squares quantization in pcm," Information Theory, IEEE Transactions on, vol. 28, no. 2, pp. 129-137, 1982.

[33] A. Laudani, F. Mancilla-David, F. Riganti-Fulginei, and A. Salvini, "Reduced-form of the photovoltaic five-parameter model for efficient computation of parameters," Solar Energy, vol. 97, pp. 122 - 127, 2013.

[34] A. Khosravi, S. Nahavandi, and D. Creighton, "Prediction intervals for short-term wind farm power generation forecasts," Sustainable Energy, IEEE Transactions on, vol. 4, no. 3, pp. 602-610, 2013.

[35] A. Saltelli, K. Chan, and E. Scott, "Sensitivity analysis wiley series in probability and statistics," Willey, New York, 2000.

[36] L. Reyes-Chamorro, A. Bernstein, J.-Y. Le Boudec, and M. Paolone, "A Composable Method for Real-Ttime Control of Active Distribution Networks with Explicit Power Setpoints. Part II: Implementation and Validation," Electric Power Systems Research, 2015.

[37] M. Arnold and G. Andersson, "Model predictive control of energy storage including uncertain forecasts," in Power Systems Computation Conference (PSCC), Stockholm, Sweden, vol. 23, 2011, pp. 24-29.

[38] F. Sossan, E. Namor, R. Cherkaoui, and M. Paolone, "Achieving the dispatchability of distribution feeders through prosumers data driven forecasting and model predictive control of electrochemical storage,' IEEE Transactions on Sustainable Energy, vol. 7, no. 4, pp. 1762-1777, Oct 2016.

[39] H. T. Pedro and C. F. Coimbra, "Assessment of forecasting techniques for solar power production with no exogenous inputs," Solar Energy, vol. 86, no. 7, pp. $2017-2028,2012$.

Enrica Scolari (S'15) was born in Italy in 1989. She received the M.Sc. (Hons.) degree in Energy Engineering from the University of Bologna, Italy, in 2014. Since 2015, she is pursuing the Ph.D. degree with the Distributed Electrical System Laboratory, Swiss Federal Institute of Technology, Lausanne, Switzerland. Her main research interests are solar photovoltaic modeling and forecasting applied to power system.

Lorenzo Reyes Chamorro (M'07) Lorenzo Reyes-Chamorro was born in Santiago, Chile in 1984. He received the B.Sc. degree in electrical engineering from the University of Chile, Santiago, Chile in 2009 and the Ph.D. degree at the Swiss Federal Institute of Technology of Lausanne (EPFL), Lausanne, Switzerland in 2016. He is currently a postdoctoral fellow at the Distributed Electrical System Laboratory (DESL), EPFL and an ad-honorem Professor at the Austral University of Chile, Valdivia, Chile.

Fabrizio Sossan (M'11) is an Italian citizen and was born in Genova in 1985. He got his M.Sc. in Computer Engineering from the University of Genova in 2010, and, in 2014, the Ph.D. in Electrical Engineering from the Danish Technical University with the thesis Indirect control of flexible demand for power system applications. Since 2015, he is a postdoctoral fellow at EPFL, Switzerland. His main research interests are modelling and optimization applied to power system.
Mario Paolone (M'07 - SM'10) was born in Italy in 1973. He received the M.Sc. (Hons.) degree in electrical engineering and the Ph.D. degree from the University of Bologna, Bologna, Italy, in 1998 and 2002, respectively. He was a Researcher of Electric Power Systems at the University of Bologna in 2005, where he was with the Power Systems Laboratory until 2011. In 2010, he was an Associate Professor with Politecnico di Milano, Milano, Italy. He is currently Professor with the Swiss Federal Institute of Technology, Lausanne, Switzerland, where he was the EOS Holding Chair of the Distributed Electrical Systems Laboratory. He has authored and co-authored more than 220 scientific papers published in reviewed journals and presented at international conferences. His current research interests include power systems with particular reference to real-time monitoring and operation, power system protections, power systems dynamics, and power system transients. Dr. Paolone is the Secretary and a member of several IEEE and Cigré Working Groups. He was the Co-Chairperson of the Technical Committee of the 9th edition of the International Conference of Power Systems Transients in 2009. He was a recipient of the IEEE EMC Society Technical Achievement Award in 2013. 